\title{
Calibration of the Accelerometer Triad of an Inertial Measurement Unit, Maximum Likelihood Estimation and Cramér-Rao Bound
}

\author{
G. Panahandeh, I. Skog, and M. Jansson \\ KTH-Royal Institute of Technology, ACCESS Linnaeus Center, Signal Processing Laboratory, Stockholm, Sweden \\ \{ghazaleh.panahandeh, isaac.skog, magnus.jansson\}@ee.kth.se
}

\begin{abstract}
In this paper, a simple method to calibrate the accelerometer cluster of an inertial measurement unit (IMU) is proposed. The method does not rely on using a mechanical calibration platform that rotates the IMU into different precisely controlled orientations. Although the IMU is rotated into different orientations, these orientations do not need to be known. Assuming that the IMU is stationary at each orientation, the norm of the input is considered equal to the gravity acceleration. As the orientations of the IMU are unknown, the calibration of the accelerometer cluster is stated as a blind system identification problem where only the norm of the input to the system is known. Under the assumption that the sensor noises have a white Gaussian distribution the system identification problem is solved using the maximum likelihood estimation method. The accuracy of the proposed calibration method is compared with the CramérRao bound for the considered calibration problem.
\end{abstract}

\section{INTRODUCTION}

Inertial navigation systems (INS) use a combination of motion sensors and rotational sensors to provide the position, orientation, and velocity of moving objects. Inertial measurement units (IMU) generally consist of three orthogonal gyroscopes and three orthogonal accelerometers. The integration of these two clusters, which provide angular velocity and specific force, yield attitude and position. Recent improvements in micro-electromechanical system (MEMS) inertial sensors allow building small, light weight, and cheap motion capture systems. The accuracy of such systems are highly dependent on the calibration of the IMU to remove the systematic errors. Calibration is the process of comparing the instruments output with a known reference information and determining the coefficients that force the output to agree with the reference information over a range of output values [1]. Using a mechanical platform for calibrating the IMU is a traditional calibration method which is economically inefficient for inlab tests. In this calibration procedure the IMU is rotated by a mechanical platform into several precisely controlled orientations. Then, the output of both the accelerometer and gyroscope clusters are observed and compared with the precalculated gravity force vector and rotational velocities, respectively, at each orientation [1], [2]. However, many times the cost of a mechanical calibration platform exceeds the cost of developing a new low-cost MEMS inertial sensor.

978-1-4244-5864-6/10\$26.00 (C) IEEE
Therefore, different calibration methods have been proposed that do not require the mechanical platform. For example, in [3], an optical tracking system is used for calibration of the IMU sensors. Furthermore, the shape from motion methods are introduced for micro force sensors resulting in rapid and effective calibration techniques [4], [5].

The six-position static is among the most commonly used fully controlled calibration method in which all the inputs, outputs and orientations are carefully set [6], [7]. This method requires the inertial system to be mounted on a leveled surface while each sensitive axis of every sensor pointing alternately up and down. Although this structure can provide good results, it seems to be costly. In [7], a multi position calibration approach is proposed. Additionally, the calibration of the inner-triad misalignment between the gyro and accelerometer is considered.

Recently, different accelerometer calibration methods have been developed utilizing the fact that for an ideal (noise free) IMU, the norm of the measured output of the accelerometer should be equal to the magnitude of the gravity force vector [6], [8], [9]. Imposing the fact in the system model of the sensors, a cost function is defined which is minimized with respect to the unknown model parameters. However, using such an assumption, the optimization of the corresponding criterion function typically leads to a biased estimate of the calibration parameters. To avoid this, we solve the system identification problem using the maximum likelihood estimation (MLE) framework. This leads to an estimator which is asymptotically unbiased. The performance of the calibration algorithm is compared with the Cramér-Rao bound. This bound sets the lower limit for the variance of the estimation error for all unbiased estimators.

The paper is organized as follows. The inertial sensor model for the accelerometer cluster is described in Section II. The proposed calibration method is described in Section III. In Section IV, the parametric Cramér-Rao bound for the system model of the accelerometer is derived. Simulation results are discussed in Section V. Finally, the conclusion of the study is summarized in Section VI. 


\section{SySTEM Model}

In the following sections scalars are denoted by lowercase letters $(\phi, \varphi)$, vectors by bold letters $(\mathbf{b}, \mathbf{u})$, and matrices by bold face capitals $(\mathbf{K}, \mathbf{R})$.

This section is divided into four subsections. First, the sensor model of the accelerometer is derived in II-A. Secondly, the input force to the system is described in II-B. Then, the unknown parameters of the system is determined in II-C. Finally, the distribution of the output is derived in II-D.

\section{A. Accelerometer Sensor Model}

As mentioned before, an IMU consists of three almost orthogonally mounted accelerometers and three almost orthogonally mounted gyroscopes; however, the sensors sensitivity axes are not orthogonal in reality, which is due to the manufacturing errors that lead to sensor axes misalignments from the orthogonal axes. The accelerometer cluster measures the linear acceleration of the system in the inertial reference frame. If the non-orthogonal sensitivity axes of the accelerometer cluster differs only by small angles from the ideal orthogonal set of platform coordinate axes, the specific force in accelerometer cluster coordinates can be transformed into specific force estimates in platform coordinates as [1]

$$
\mathbf{s}^{p}=\mathbf{T}_{a}^{p} \mathbf{s}^{a}, \quad \mathbf{T}_{a}^{p}=\left(\begin{array}{ccc}
1 & -\alpha_{y z} & \alpha_{z y} \\
\alpha_{x z} & 1 & -\alpha_{z x} \\
-\alpha_{x y} & \alpha_{y x} & 1
\end{array}\right)
$$

where $\mathbf{s}^{p}$ and $\mathbf{s}^{a}$ denote the specific force in platform and accelerometer coordinates, respectively [8]. In (1), $\alpha_{i j}$ is the rotation of the $i$-th accelerometer sensitivity axis around the $j$-th platform axis. Defining the platform coordinate system so that the platform coordinate axis $x^{p}$ coincides with the $x^{a}$ accelerometer sensitivity axis, and $y^{p}$ axis lies in the plane spanned by $x^{a}$ and $y^{a}$, then the angles $\left\{\alpha_{x z}, \alpha_{x y}, \alpha_{y x}\right\}$ become zero. That is, (1) is reduced to

$$
\mathbf{s}^{p}=\mathbf{T}_{a}^{p} \mathbf{s}^{a}, \quad \mathbf{T}_{a}^{p}=\left(\begin{array}{ccc}
1 & -\alpha_{y z} & \alpha_{z y} \\
0 & 1 & -\alpha_{z x} \\
0 & 0 & 1
\end{array}\right) .
$$

The sensor bias is another source of the error in the accelerometer cluster, which is the observed signal when no input is present. Moreover, this is an acceleration-insensitive error that can be defined as

$$
\mathbf{b}_{a}=\left[\begin{array}{lll}
b_{x} & b_{y} & b_{z}
\end{array}\right]^{T}
$$

where $b_{i}$ is the unknown bias of the $i$-th accelerometer output and $[.]^{T}$ denotes the transpose operation. The accelerometer scale factor error, which is the ratio of the change in output (in volts or amperes) to a unit change of the input (in units of acceleration), is represented by the scale factor matrix $\mathbf{K}_{a}$ as

$$
\mathbf{K}_{a}=\left(\begin{array}{ccc}
k_{x} & 0 & 0 \\
0 & k_{y} & 0 \\
0 & 0 & k_{z}
\end{array}\right)
$$

where $k_{i}$ is the unknown scaling of the $i$-th accelerometer output. Based on (2), (3), and (4), the model of the measured output of the accelerometer cluster is

$$
\tilde{\mathbf{s}}^{a}=\mathbf{K}_{a}\left(\mathbf{T}_{a}^{p}\right)^{-1} \mathbf{s}^{p}+\mathbf{b}_{a}+\mathbf{v}_{a}
$$

where $\mathbf{s}^{p}$ denotes the input force expressed in the platform coordinates and $\mathbf{v}_{a}$ is a noise term reflecting the measurement noise from the sensor [1].

Calibration of the accelerometer cluster by using a mechanical platform has been done by rotating the cluster into different precisely controlled orientations and angular rates. Then, the output of the accelerometer cluster is compared with the precalculated specific force to estimate the nine calibration parameters, including the three scale factors, the three misalignment angles, and three biases:

$$
\theta_{1}=\left[\begin{array}{lllllllll}
k_{x} & k_{y} & k_{z} & \alpha_{y z} & \alpha_{z y} & \alpha_{z x} & b_{x} & b_{y} & b_{z}
\end{array}\right]^{T} .
$$

Using such an expensive mechanical platform for the calibration procedure of a low cost MEMS sensor is not economically efficient while it costs many times more than the cost of a new sensor. Our proposed method does not rely on using a mechanical calibration platform even though the IMU is rotated in different random orientations.

For the sake of simplicity, we will skip the subscripts and superscripts, $a$ and $p$ in the following. In general, the signal in noise model of the measured output of the accelerometer cluster based on (5) can be written as

$$
\mathbf{y}_{k}=\mu_{k}+\mathbf{v}_{k}
$$

where $\mathbf{y}_{k}$ is the output at the $k$-th measurement and $\mathbf{v}_{k}$ is zero mean white Gaussian noise with variance $\sigma^{2}$; the signal model $\mu_{k}$ is defined as

$$
\mu_{k}=\mu\left(\theta_{1}, \mathbf{u}_{k}\right)=\mathbf{K T}^{-1} \mathbf{u}_{k}+\mathbf{b}
$$

where $\mathbf{u}_{k}$ is the input force at the $k$-th measurement, corresponding to the specific force $\mathbf{s}^{p}$, and $\theta_{1}$ is the parameter vector (6).

\section{B. Input Force}

Because accelerometers in the IMU sensors are used in the cluster of components, using a reference vector acquired from geodetic surveys that can be used for calibration of all the three components at the same time is of interest [1]. A suitable reference specific force for the accelerometer cluster calibration is the gravity acceleration. Assuming that the IMU is stationary at each orientation and the only force to the system is gravity acceleration, the norm of the input is considered equal to the gravity acceleration. In fact, the acceleration input force in the platform coordinate is related to the navigation-frame gravity vector by the rotation matrix $\mathbf{R}_{n}^{p}$, Fig. 1. The rotation matrix can be defined by a series of three plane rotations involving the Euler angles $(\varphi, \phi, \psi)=($ roll, pitch, and heading) [10]:

$$
\mathbf{R}_{n}^{p}=\left(\begin{array}{ccc}
c \psi c \phi & s \psi c \phi & -s \phi \\
-s \psi c \phi+c \psi s \phi s \varphi & c \psi c \varphi+s \psi s \phi s \varphi & c \phi s \varphi \\
s \psi s \phi+c \psi s \phi c \varphi & -c \psi s \varphi+s \psi s \phi c \varphi & c \phi c \varphi
\end{array}\right)
$$




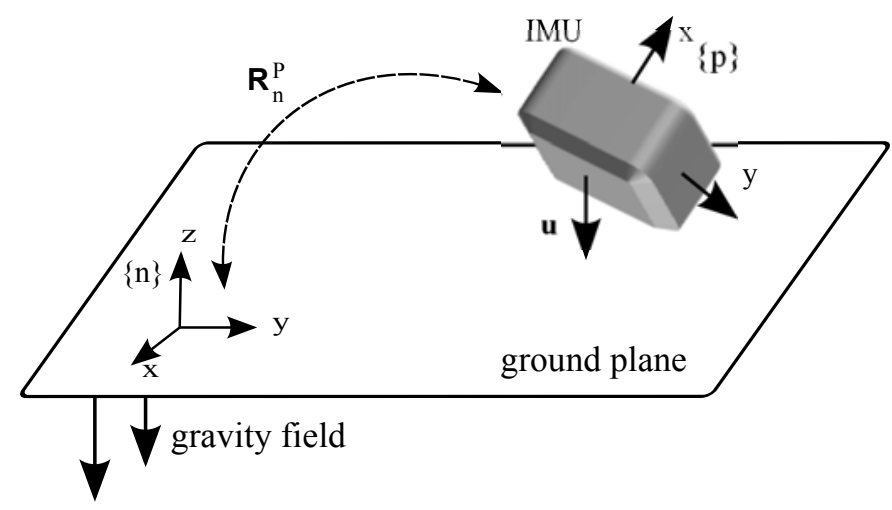

Fig. 1. The geometric relation between the IMU platform coordinate frame $\{\mathrm{p}\}$, and navigation frame $\{\mathrm{n}\}$.

where c stands for cosine and s stands for sine functions. Thus, the input force to the system is

$$
\begin{aligned}
\mathbf{u}_{k} & =\mathbf{R}_{n}^{p} \mathbf{g} \\
& =\mathbf{R}_{n}^{p}\left(\begin{array}{c}
0 \\
0 \\
-g
\end{array}\right) \\
& =-\left(\begin{array}{c}
-s \phi \\
c \phi s \varphi \\
c \phi s \varphi
\end{array}\right) g
\end{aligned}
$$

where $g$ is the magnitude of the apparent gravity vector.

\section{Unknown Parameters}

The nine-parameter sensor model of the accelerometer cluster is considered (6), (7), and (8); the three scale factors, the three misalignment angles, and three biases. To estimate these unknown calibration parameters, the IMU is required to be rotated in different orientations. Each time the IMU is placed in a new orientation, two new unknown rotational angles appear in the input force $\mathbf{u}_{k}$ to the system; however, the norm of the $\mathbf{u}_{k}$ is equal to the magnitude of the gravity force. In order to estimate the calibration parameters $\theta_{1}$, these unknown Euler angles need to be simultaneously estimated together with the calibration parameters. Define the parameter vector $\theta_{2}$, which collects all the unknown Euler angles, as:

$$
\theta_{2}=\left[\begin{array}{lllllll}
\phi_{1} & \varphi_{1} & \phi_{2} & \varphi_{2} & \ldots & \phi_{M-1} & \varphi_{M-1}
\end{array}\right]^{T}
$$

where $M$ is the number of orientations that the IMU is placed in. We are not really interested in these rotation angles and they serve only to complicate the problem. In some estimation approaches, we can get rid of estimating these parameters (known as nuisance parameters) [11]. The uncertainty in the rotational angles which appears in the input force of the system makes our calibration problem a blind identification problem. To deal with these nuisance parameters, a new vector of parameters, $\theta$, is introduced which is the combination of the calibration parameter vector and the Euler angle parameter vector:

$$
\theta=\left[\begin{array}{ll}
\theta_{1}^{T} & \theta_{2}^{T}
\end{array}\right]^{T}
$$

To estimate the unknown parameter vector $\theta$, the IMU is rotated into several random orientations. Because the number of calibration parameters are nine, the minimum number of rotations that is needed to estimate $\theta_{1}$ is nine, $M \geq 9$. Increasing the number of rotations, more accurate results can be achieved, although more unknown parameters will appear in $\theta_{2}$.

\section{Output Distribution}

Collecting all the measurement outputs and signal models into bigger vectors, the total measurement output $\widetilde{\mathbf{y}}$, and the signal model $\widetilde{\mu}$ are defined as:

$$
\begin{aligned}
\widetilde{\mathbf{y}} & =\left[\begin{array}{llll}
\mathbf{y}_{1}^{T} & \mathbf{y}_{2}^{T} & \ldots & \mathbf{y}_{M N}^{T}
\end{array}\right]^{T} \\
\widetilde{\mu} & =\left[\begin{array}{llll}
\mu_{1}^{T} & \mu_{2}^{T} & \ldots & \mu_{N M}^{T}
\end{array}\right]^{T}
\end{aligned}
$$

where $N$ is the number of samples which are taken in each rotation. Based on the signal in noise model (7), the output signal, $\mathbf{y}_{k}$, has a Gaussian distribution as

$$
\mathbf{y}_{k} \sim N\left(\mu_{k}, \sigma^{2} \mathbf{I}_{3}\right)
$$

Thus, the probability density function of the measurement $\widetilde{\mathbf{y}}$ is

$$
p(\widetilde{\mathbf{y}} ; \theta)=\frac{1}{(2 \pi)^{\frac{M N}{2}}|\mathbf{C}(\theta)|^{\frac{1}{2}}} \exp \left(\frac{-1}{2 \sigma^{2}} \sum_{k=1}^{N M}\left\|\mathbf{y}_{k}-\mu_{k}\right\|^{2}\right)
$$

where

$$
\mathbf{C}(\theta)=\sigma^{2} \mathbf{I}_{3 M N}
$$

$\mathbf{C}(\boldsymbol{\theta})$ is the covariance matrix of $\widetilde{\mathbf{y}}, \mathbf{I}$ is the identity matrix with the subscripted dimension, $|\cdot|$ denotes the determinant, and $\|\cdot\|$ refers to the Euclidean length of a vector.

\section{PROPOSED CALIBRATION METHOD}

Assuming, the sensor measurements are disturbed by additive white Gaussian noise, the observed data has a multivariate Gaussian distribution and the mean vector contains all the unknown parameters to be estimated. To derive an asymptotically unbiased estimator, a maximum likelihood estimation (MLE) framework is used. Since finding a closed form, one step, solution for maximizing the likelihood function in this identification problem is difficult, an iterative approach is proposed. In our method, the unknown parameters are clustered into two groups, the rotational angles and the calibration parameters. The optimization is done by maximizing the likelihood function with respect to one group of parameters at a time while keeping the remaining parameters fixed at their current values.

In order to estimate the parameter vector $\theta$, it is typical to introduce the log-likelihood function defined as

$$
L(\theta)=\ln p(\tilde{\mathbf{y}} ; \theta) .
$$

The likelihood function is a function of the parameter vector $\theta$ given the data $\tilde{\mathbf{y}}$. The idea is to find $\theta$ such that $p(\tilde{\mathbf{y}} ; \theta)$ is a maximum. The method of maximum likelihood estimates $\hat{\theta}$ 
by finding the value of $\theta$ which maximizes the log-likelihood function or equivalently minimizes

$$
J(\theta)=\sum_{k=1}^{N M}\left\|\mathbf{y}_{k}-\mu_{k}\right\|^{2}
$$

Because $\mu$ is a nonlinear function of $\theta$, the minimization of $J$ becomes difficult. One way to reduce the complexity of this problem is to use separability of the parameters [11]. The separable signal model of the system may be formed by rewriting (8) as

$$
\mu_{k}=\mathbf{H}\left(\theta_{1}\right)\left(\begin{array}{c}
\mathbf{u}_{k} \\
1
\end{array}\right)_{4 \times 1}, \quad \mathbf{H}\left(\theta_{1}\right)=\left(\mathbf{K T}^{-1} \mid \mathbf{b}\right)
$$

here, $(\mathbf{A} \mid b)$ denotes extending the matrix $\mathbf{A}$ with a column vector $\mathbf{b}$. As a result, the minimization is performed in two steps; First step: minimize $J$ with respect to $\theta_{2}$, Second step: replacing the estimated value for $\theta_{2}$ in $J$, and minimize it with respect to $\theta_{1}$. In the following, details of these two steps are described.

\section{A. First Step}

Based on the signal model (18), the minimization of $J(\theta)$ over the parameter vector $\theta_{2}$ can be performed to get $\hat{\theta}_{2}$ :

$$
\hat{\boldsymbol{\theta}}_{2}=\underset{\theta_{2}}{\arg \min } \sum_{k=1}^{N M}\left\|\mathbf{y}_{k}-\mathbf{H}\left(\boldsymbol{\theta}_{1}\right)\left(\begin{array}{c}
\mathbf{u}_{k} \\
1
\end{array}\right)\right\|^{2} .
$$

It can be proved from equation (10) that this is a constrained minimization problem because any solution must satisfy $\left\|\mathbf{u}_{k}\right\|=g$. Since the input force is constant during the $N$ samples and Euler angles, $\phi_{i} \mathrm{~s}$ and $\varphi_{i} \mathrm{~s}$, are independent at each rotation, the minimization in (19) breaks down into $M$ independent minimization problems for each rotation $i$ $(i=1, \ldots, M)$ as

$$
\begin{gathered}
\left(\hat{\phi}_{i}, \hat{\varphi}_{i}\right)=\underset{\phi_{i}, \varphi_{i}}{\arg \min } \sum_{j=1}^{N}\left\|\mathbf{y}_{i, j}-\mathbf{H}\left(\theta_{1}\right)\left(\begin{array}{c}
\mathbf{u}_{i} \\
1
\end{array}\right)\right\|^{2} \\
\text { s.t }\left\|\mathbf{u}_{i}\right\|=g
\end{gathered}
$$

and

$$
\hat{\theta}_{2}=\left[\begin{array}{lllllll}
\hat{\phi}_{1} & \hat{\varphi}_{1} & \hat{\phi}_{2} & \hat{\varphi}_{2} & \ldots & \hat{\phi}_{M} & \hat{\varphi}_{M}
\end{array}\right]^{T}
$$

where $\mathbf{y}_{i, j}$ is the $j$-th measurement output of the accelerometer cluster at the $i$-th rotation. Because of the given constraint, the minimization problem expressed in (20) is not a convex problem; therefore, it can not be solved by common convex optimization algorithms. Consequently, a nonlinear least square curve fitting method is used to estimate the unknown Euler angles. Moreover, to start the iterations an appropriate initial value for the calibration parameters should be considered. Starting from the ideal case, when all the source of the errors are assumed to be removed, has been found to produce good results.

\section{B. Second Step}

Once $\theta_{2}$ is estimated, the minimization is continued by finding $\hat{\theta}_{1}$ :

$$
\hat{\theta}_{1}=\underset{\theta_{1}}{\arg \min } \sum_{k=1}^{N M}\left\|\mathbf{y}_{k}-\mathbf{H}\left(\theta_{1}\right)\left(\begin{array}{c}
\hat{\mathbf{u}}_{k} \\
1
\end{array}\right)\right\|^{2} .
$$

One solution to estimate $\theta_{1}$ is through the linear least squares estimation approach. This is because $\mathbf{H}\left(\theta_{1}\right)$ can be reparameterized to depend linearly on a new set of parameters.

This two-step minimization procedure is iterated until a local maximum of the likelihood is attained.

\section{CRAMÉR-RAO BOUND FOR THE PARAMETRIC MODEL}

When evaluating the performance of an estimator often it is of interest to compare the obtained estimation error with an optimal (unbiased with minimum variance) estimator. The optimal estimator may not exist, be unknown, or too complex to obtain; however, the performance of the optimal estimator only depends on the properties of the signal model, and may therefore be calculated independently. The Cramér-Rao bound sets the lower limit for the variance of the estimation error for all unbiased estimators [11].

The parametric Cramér-Rao bound under the regularity condition is defined as

$$
\operatorname{var}(\hat{\boldsymbol{\theta}}(i)) \geq\left[\mathbf{I}^{-1}(\boldsymbol{\theta})\right]_{i i}
$$

where the left hand side is the variance of the $i$-th element of $\hat{\theta}, \mathbf{I}(\theta)$ is the Fisher information matrix, and $[\cdot]_{i j}$ indicates $i j$ th element of the given matrix. The Fisher information matrix for the general Gaussian case is given by

$$
\begin{aligned}
& {[\mathbf{I}(\theta)]_{i j}=\left[\frac{\partial \mu(\theta)}{\partial \theta(i)}\right]^{T} \mathbf{C}^{-1}(\theta)\left[\frac{\partial \mu(\theta)}{\partial \theta(j)}\right]} \\
& +\frac{1}{2} \operatorname{tr}\left[\mathbf{C}^{-1}(\theta) \frac{\partial \mathbf{C}(\theta)}{\partial \theta(i)} \mathbf{C}^{-1}(\theta) \frac{\partial \mathbf{C}(\theta)}{\partial \theta(j)}\right]
\end{aligned}
$$

Assuming we know the variance of the noise $\sigma^{2}$, (24) is simplified to

$$
[\mathbf{I}(\theta)]_{i j}=\frac{1}{\sigma^{2}}\left[\frac{\partial \mu(\theta)}{\partial \theta(i)}\right]^{T}\left[\frac{\partial \mu(\theta)}{\partial \theta(j)}\right] .
$$

Using the fact that $\mathbf{u}_{k}$ is constant for all the $N$ samples at each orientation, the Fisher information matrix may be written as

$$
[\mathbf{I}(\theta)]_{i j}=\frac{N}{\sigma^{2}} \sum_{m=1}^{M}\left[\frac{\partial \mu_{m}}{\partial \theta(i)}\right]^{T}\left[\frac{\partial \mu_{m}}{\partial \theta(j)}\right]
$$

By simplifying (26), the Fisher information matrix can be written in a block matrix form as

$$
\mathbf{I}(\theta)=\left(\begin{array}{cc}
\mathbf{I}_{1}\left(\theta_{1}\right)_{9 \times 9} & \mathbf{K} \\
\mathbf{K}^{T} & \mathbf{I}_{2}\left(\boldsymbol{\theta}_{2}\right)_{2 M \times 2 M}
\end{array}\right)
$$

where $\mathbf{I}_{i}\left(\theta_{i}\right)$ is the Fisher information matrix, considering only $\theta_{i}$ as the unknown parameter vector. Using Sherman-Morrison formula, the inverse of the first block of $\mathbf{I}(\theta)$ which leads to the 


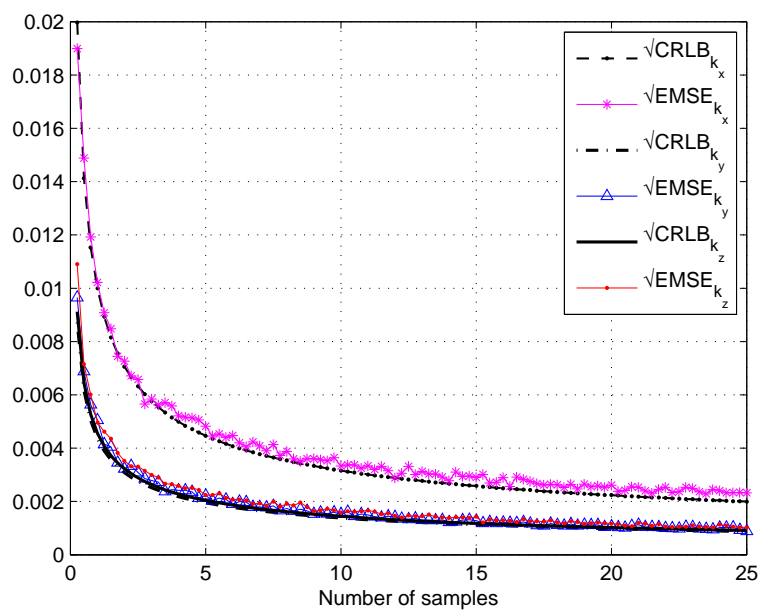

Fig. 2. Square-root of the empirical mean square errors (EMSE) of the estimated scale-factors and the square-root of the Cramér-Rao bounds (CRLB), versus the number of samples at each orientation.

parametric Cramér-Rao bound for the calibration parameters can be found as:

$$
\operatorname{var}\left(\hat{\boldsymbol{\theta}}_{1}(i)\right) \geq\left[\mathbf{I}_{1}\left(\theta_{1}\right)^{-1}+\mathbf{I}_{1}\left(\theta_{1}\right)^{-1} \mathbf{K E}^{-1} \mathbf{K}^{T} \mathbf{I}_{1}\left(\theta_{1}\right)^{-1}\right]_{i i}
$$

where

$$
\mathbf{E}=\mathbf{I}_{2}\left(\theta_{2}\right)-\mathbf{K}^{T} \mathbf{I}_{1}\left(\theta_{1}\right)^{-1} \mathbf{K}
$$

\section{Simulation RESULTS}

The proposed calibration approach has been evaluated by Monte-Carlo simulations. In the presented simulation, the IMU was rotated into nine unknown orientations and the accelerometer noise variance was set to $0.01\left[\mathrm{~m} / \mathrm{s}^{2}\right]$. The empirical mean square errors of the estimated calibration parameters along with the corresponding Cramér-Rao bounds are shown in Fig. 2-4. These results are obtained from 500 Monte-Carlo simulations, when the IMU is rotated into nine different unknown angles (the least number of rotations) and in each orientation 25 samples are taken. The simulation results show that the square-root of the empirical mean square error (EMSE) of the estimated parameters converges to the squareroot of the Cramér-Rao bound already after a few numbers of measurements.

The average value and standard deviation of the estimated calibration parameters are summarized in Table I. The results are calculated for 100 Monte-Carlo simulations, while IMU is rotated into 25 different orientations and at each orientation the sensor is sampled 25 times.

\section{CONCLUSION}

An approach for calibrating the accelerometer cluster of IMUs has been proposed. The method does not require any expensive mechanical calibration platform. Instead, the proposed calibration method is based on a maximum likelihood estimator that jointly estimates the orientations of the IMU and the calibration parameters of interest. Considering the sensors

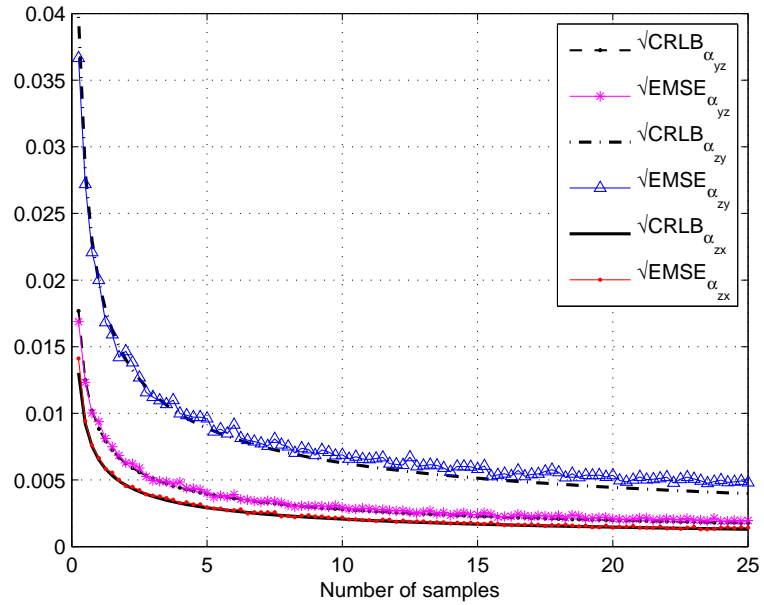

Fig. 3. Square-root of the empirical mean square errors (EMSE) of the estimated misalignments and the square-root of the Cramér-Rao bounds (CRLB), versus the number of samples at each orientation.

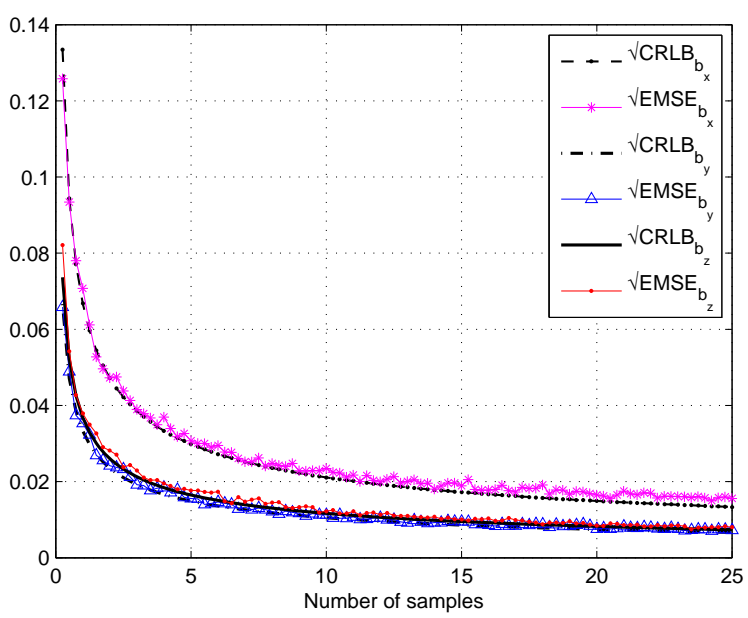

Fig. 4. Square-root of the empirical mean square errors (EMSE) of the estimated biases and the square-root of the Cramér-Rao bounds (CRLB), versus the number of samples at each orientation.

TABLE I

SIMULATION RESULT FOR THE ACCELEROMETER CLUSTER OF AN IMU FOR $N=M=25$.

\begin{tabular}{lcccc}
\hline \hline Calibration parameter & True value & Average value & Standard deviation \\
\hline \multirow{4}{*}{ Scale factors } & $k_{x_{a}}$ & 1.05 & 1.0499 & 0.0012 \\
& $k_{y_{a}}$ & 0.93 & 0.9299 & 0.0009 \\
& $k_{z_{a}}$ & 1.06 & 1.0600 & 0.0013 \\
\hline \multirow{3}{*}{ Misalignment $\left[^{\circ}\right]$} & $\alpha_{y z}$ & 2 & 2.0029 & 0.0018 \\
& $\alpha_{z y}$ & -5 & -5.0089 & 0.0024 \\
& $\alpha_{z x}$ & 3 & 3.0180 & 0.0017 \\
\hline \multirow{3}{*}{ Biases $\left[\mathrm{m} / \mathrm{s}^{2}\right]$} & $b_{x_{a}}$ & 0.32 & 0.3207 & 0.0095 \\
& $b_{y_{a}}$ & 0.63 & 0.6294 & 0.0070 \\
\hline \hline
\end{tabular}


parameters and rotational angles, a closed form expression for Cramér-Rao bound is derived and results are compared with this theoretical bound. The simulation results show that the empirical mean square error (EMSE) of the sensor parameters estimated using the proposed calibration method converges to the Cramér-Rao bound after only a few measurements.

\section{REFERENCES}

[1] A. Chatfield, Fundamentals of High Accuracy Inertial Navigation. American Institute of Aeronautics and Astronautics, 1997.

[2] J. Hung, J. Thacher, and H. White, "Calibration of accelerometer triad of an imu with drifting $\mathrm{z}$-accelerometer bias," in Proc. IEEE National, Aerospace and Electronics Conf., pp. 153 -158 vol.1, May. 1989.

[3] A. Kim and M. Golnaraghi, "Initial calibration of an inertial measurement unit using an optical position tracking system," in Proc. Position Location and Navigation Symposium, pp. 96 -101, Apr. 2004.

[4] K. Kim, Y. Sun, R. M. Voyles, and B. J. Nelson, "Calibration of multiaxis mems force sensors using the shape-from-motion method," IEEE Sensors Journal, vol. 7, pp. 344 -351, March 2007.

[5] M. Hwangbo and T. Kanade, "Factorization-based calibration method for mems inertial measurement unit," in Proc. IEEE Int. Conf. on Robotics and Automation, pp. 1306-1311, May. 2008.

[6] Z. F. Syed, P. Aggarwal, C. Goodall, X. Niu, and N. El-Sheimy, "A new multi-position calibration method for mems inertial navigation systems," Measurement Science and Technology, vol. 18, pp. 1897 -1907, May 2007.

[7] H. Zhang, Y. Wu, W. Wu, M. Wu, and X. Hu, "Improved multi-position calibration for inertial measurement units," Measurement Science and Technology, vol. 21, pp. 15107-15117(11), Jan. 2010.

[8] I. Skog and P. Händel, "Calibration of a MEMS inertial measurement unit," in Proc. IMEKO XVIII World Congress, Sep. 2006.

[9] I. Frosio, S. Stuani, and N. Borghese, "Autocalibration of mems accelerometer," in Proc. IEEE Instrumentation and Measurement Technology Conf., pp. 519 -523, Apr. 2006.

[10] J. A. Farrell and M. Barth, Global Positioning System, Inertial Navigation and Integration. McGraw-Hill Companies, 1999.

[11] S. M. Kay, Fundamentals of Statistical Signal Processing, Estimation Theory. Prentice Hall, 1999. 